\title{
Research on Exchange Rate Forecasting Model Based on ARIMA Model and Artificial Neural Network Model
}

\author{
XU Min ${ }^{1, a}$ and LI Weiguo ${ }^{1, b}$ \\ ${ }^{1}$ Beihang University, School of Mathematics and System Science, \\ No.37, College Road, \\ Haidian District, Beijing, China \\ axusparrow@outlook.com, ${ }^{\mathrm{b}}$ liwg@buaa.edu.cn
}

Keywords: ARIMA model, BP neural network model, exchange rate

\begin{abstract}
This paper uses the ARIMA and BP neural network model to forecast the exchange rate by collecting the monthly exchange rate data of RMB exchange rate from 2001 to 2017, and put forward the combined forecasting method of ARIMA and BP neural network model. From the prediction results, the results of the combined forecast are obviously better than the single model. The prediction error of the combined prediction model is 0.1433 , while the prediction error of ARIMA and BP single model is 0.1514 and 0.1677.
\end{abstract}

\section{Introduction}

With the development of the global economy, foreign exchange rate has become an important criterion for measuring international economic relations. For the exchange rate forecasting model most methods use ARIMA forecasting method. However, the ARIMA model itself is a linear model and can't be measured and predicted for non-linear parts of the exchange rate. The artificial neural network model has a good prediction for the nonlinear part. In this paper, ARIMA model and artificial neural network model are established for exchange rate data respectively, and a combined forecasting method based on two models is proposed.

\section{ARIMA Model}

The ARIMA model is established by first converting the non-stationary time series into a stationary time series by means of differential method. Then, by selecting the minimum model BIC value and other methods to determine the value of the dependent variable and the value of the hysteresis of the random error term. Finally build the model.

There are four steps to establishing the ARIMA model and forecasting. First, the time series is stabilized by the difference and the order of the difference $d$ is determined. Secondly, by observing the autocorrelation coefficient and the partial autocorrelation coefficient, the parameters of different 
orders are modeled according to the BIC minimum criterion to determine the order of the model $p$, q. Again, estimate the parameters of the model, and conduct a residual test to determine whether the established model is passed. Finally, the model is modeled using the appropriate parameters.

\section{BP Neural Network Model}

The artificial neural network consists of the basic neuron model, and the multiple neurons connect to the multi-layer network. The multi-layer network needs the BP algorithm to solve the parameters.

BP algorithm is the most widely used algorithm, which is the abbreviation of error back propagation algorithm. Based on the gradient descent method, the cumulative error on the training set is minimized, and the connection weight is continuously adjusted, and the appropriate model is obtained.

\section{The model in the exchange rate forecast application}

Data sources and basic analysis. The data comes from Canada Pacific Exchange Rate Service (http://fx.sauder.ubc.ca). This paper takes January 2000 to April 2017 the exchange rate of the RMB exchange rate as a general data set. The total data set has 196 USD exchange rate monthly data, no missing value, which will be January 2000 to December 2015 data as a training set. The data from January 2016 to April 2017 are used as test set.

The time series of the data for the training set is shown in Figure 1.

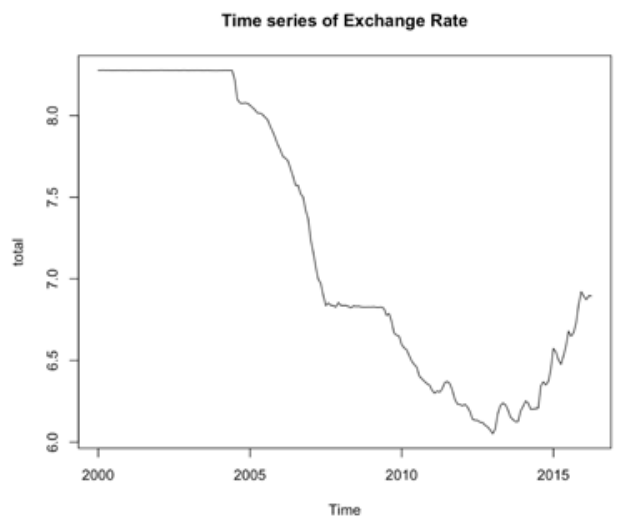

Fig.1: Time series of exchange rate

It can be seen from Figure 1, from 2000 to around 2013, the dollar exchange rate of the RMB is gradually smaller with time. From 2000 to 2005 or so, the exchange rate remained unchanged at around 8.3. From 2006 to 2010, the exchange rate began rapid decline, has stabilized at around 6.8 and 6.3, this rapid decline may be the outbreak of the US economic crisis in 2007, the economic 
crisis makes the dollar began to depreciate, the exchange rate fluctuations. Since 2013, the exchange rate changes were random and there was an upward trend.

Establish ARIMA model. The first order difference and the second order difference of the training set data are used to observe the change of the autocorrelation coefficient of the data set at different differences, and the difference degree of the self-correlation coefficient is chosen as the difference of the data. According to the comparison, choose the second order difference is more appropriate. The time series and the autocorrelation coefficients of the first order difference and the second order difference are shown in Figure 2 and Figure 3.
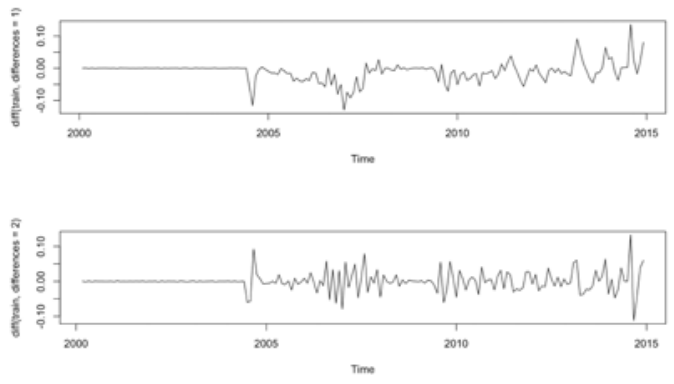

Fig.2: Differential time series
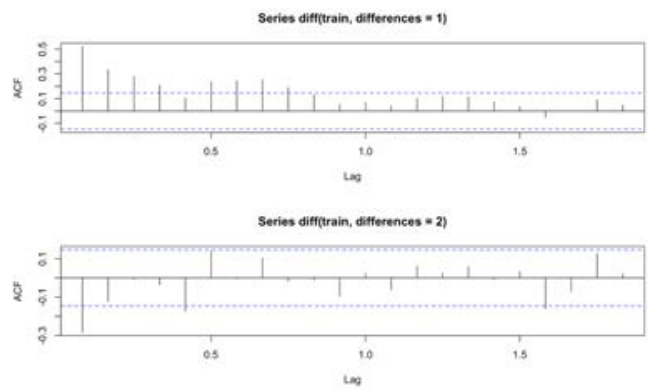

Fig.3: Difference autocorrelation coefficient graph

Therefore, the difference between the order should choose the second order difference is more appropriate, that is, $d=2$.

Try various ARIMA models for different $p$ and $q$, compare the BIC criteria of each model, selecting the BIC smaller model, for the optimal model. Make a hot chart of the BIC, as shown in Figure 4.

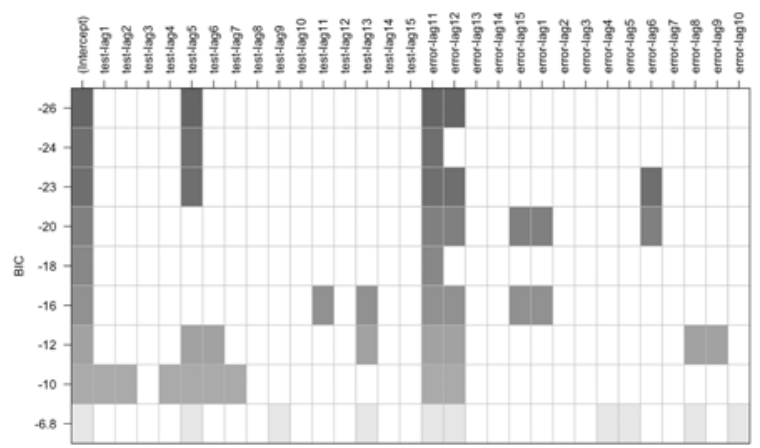

Fig.4: BIC values for each ARIMA model 
As can be seen from Figure 5, the model that makes the BIC smallest is ARIMA $(5,2,11)$.The model was diagnosed to plot the p-value (dashed line 0.05), the residual acf () and pacf () plot of the Ljung-Box test with lagged from 1 to 30, as shown in Figure 5.

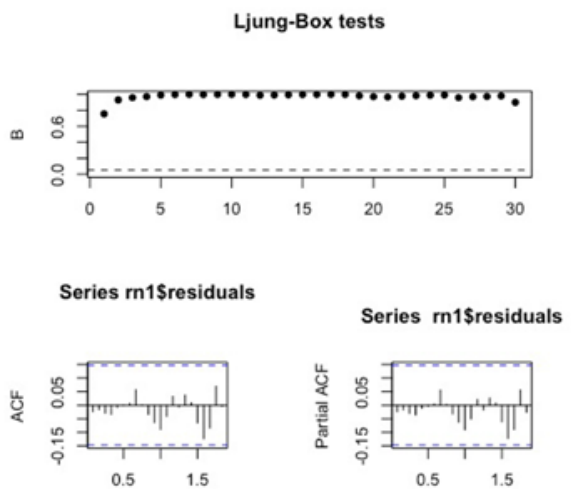

Fig.5: Ljung-Box diagram

As can be seen from Figure 6, the ARIMA $(5,2,11)$ model is tested by residuals.

Establish BP Neural Network Model. According to ARIMA order 5, select the input is five values. The output is a value. By establishing the artificial neural network model with different hidden layer nodes, the observation error is the smallest to determine the appropriate hidden layer nodes, as shown in Figure 6.

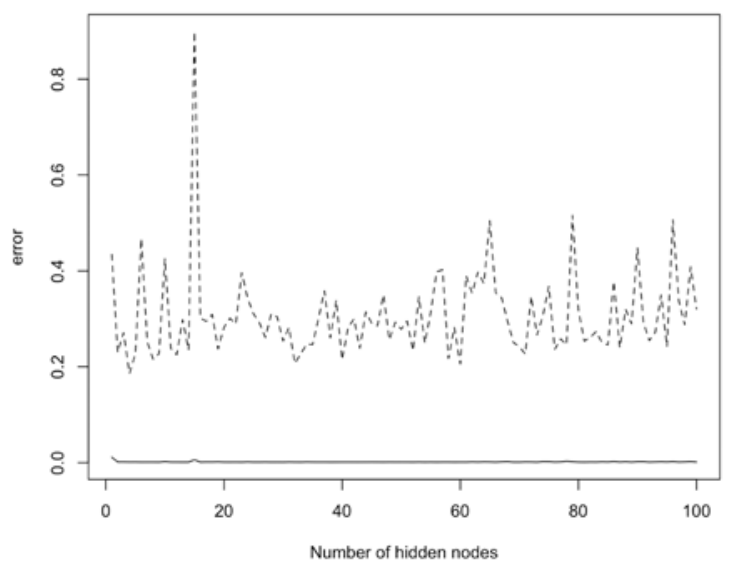

Fig.6: Hidden layer node selection

It can be seen from Figure 7 that it is appropriate to select 25 hidden nodes. The data were normalized, and the model was solved by the gradient descent method. The number of training times was set to 1000 times, and the artificial neural network model was established. 


\section{Model prediction and comparison}

The data from January 2016 to April 2017 are selected as the test sets, and the two models are used to predict each other. The relative prediction error (RPE) is used to measure the quality of the model.

$$
\mathrm{RPE}=\frac{1}{\mathrm{~N}_{\text {test }}} \frac{\sum_{\mathrm{t} \in \mathrm{test}}\left(\mathrm{r}_{\mathrm{t}}^{\text {pre }}-\mathrm{r}_{\mathrm{t}}^{\text {true }}\right)^{2}}{\operatorname{var}\left(\mathrm{r}_{\mathrm{t}}^{\text {true }}\right)}
$$

The relative prediction error of ARIMA model is 0.1514, and the prediction error of BP neural network is 0.1677 . The prediction error of the two models is weighted by the prediction error. The weight selection is based on the variance of the data, and the prediction error of the combined model is 0.1433 . The prediction results and errors of the three models are shown inTable1 and Figure 7 , the data is normalized.

Table1: Normalized data prediction comparison

\begin{tabular}{|c|c|c|c|c|}
\hline (Normalized) & True & ARIMA & $\mathrm{BP}$ & ARIMA\&BP \\
\hline 2016.01 & 0.2348 & 0.1903 & 0.1903 & 0.1903 \\
\hline 02 & 0.2246 & 0.2759 & 0.2483 & 0.2628 \\
\hline 03 & 0.2031 & 0.2264 & 0.2241 & 0.2253 \\
\hline 04 & 0.1911 & 0.2013 & 0.2221 & 0.2112 \\
\hline 05 & 0.2145 & 0.1834 & 0.1907 & 0.1869 \\
\hline 06 & 0.2427 & 0.2300 & 0.2059 & 0.2185 \\
\hline 07 & 0.2820 & 0.2519 & 0.2280 & 0.2406 \\
\hline 08 & 0.2785 & 0.3110 & 0.2860 & 0.2991 \\
\hline 09 & 0.3058 & 0.2669 & 0.2704 & 0.2686 \\
\hline 10 & 0.3567 & 0.2855 & 0.3043 & 0.2945 \\
\hline 11 & 0.3909 & 0.3224 & 0.3058 & 0.3145 \\
\hline 12 & 0.3782 & 0.3772 & 0.3529 & 0.3657 \\
\hline 2017.01 & 0.3686 & 0.4100 & 0.3930 & 0.4019 \\
\hline 02 & 0.3795 & 0.3817 & 0.3878 & 0.3846 \\
\hline 03 & 0.3787 & 0.3769 & 0.3776 & 0.3772 \\
\hline RPE & & 0.1677 & 0.1514 & 0.1433 \\
\hline
\end{tabular}




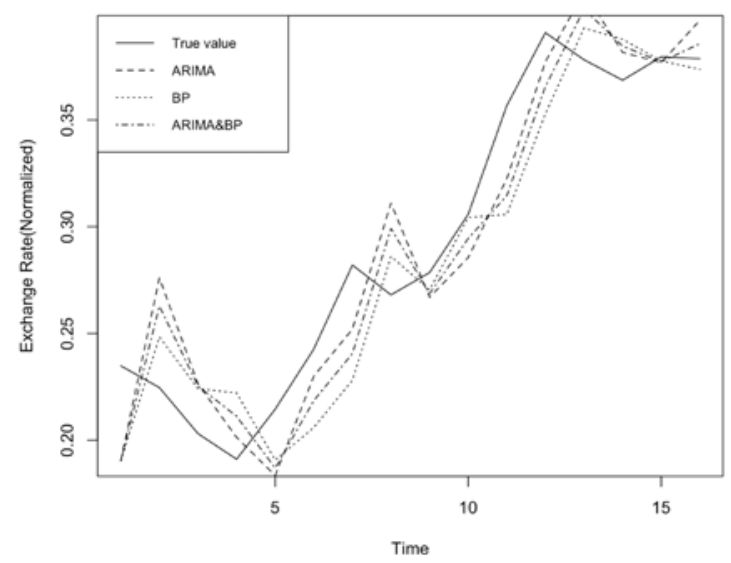

Fig.7: Prediction of different models

It can be seen from Figure 8 that the ARIMA model and the BP neural network model are difficult to distinguish between the pros and cons of the BP neural network in 2016, and the ARIMA model from January 2016 to January 2017 has better prediction results, And the combined model balance the advantages of the two models, the prediction results are smoother, and the prediction error is the smallest, and can be used as the final model.

\section{Conclusion}

This paper uses the BP neural network and the ARIMA model to predict the exchange rate of the US dollar. This method can describe the linear law of data, but also describe the nonlinear law. From the prediction results, the comprehensive forecast also has smaller prediction error, so the model has some practical significance.

\section{References}

[1] Anastasakis L. Mort N. Exchange rate forecasting using a combined parametric and nonparametric self-or ganising modeling approach :Expert Systems with Applications.(2009)

[2] Arena P, Caponetto R Fortuna L, et al.Chaos in a Fractional Order Duffing System in: Proceedings ECCTD, Budapest.(1997)

[3] Brooks C. , Testing for non-linearity in daily streling exchange rates: Applied Finance Economics.(1986)

[4] Khashei M. and M. , Bijari M, Ardali GAR. Improvement of auto-regressive integrated moving average models using fuzzy logic and artificial neural networks: Neurocomputing.(2009) 